\title{
Design Space Approach for Preservative System Optimization of an Anti-Aging Eye Fluid Emulsion
}

Felipe Rebello Lourenço ${ }^{1}$, Fabiane Lacerda Francisco ${ }^{1}$, Márcia Regina Spuri Ferreira ${ }^{1}$, Terezinha de Jesus Andreoli Pinto $^{1}$, Raimar Löbenberg ${ }^{2}$, Nádia Bou-Chacra ${ }^{1}$

${ }^{1}$ Department of Pharmacy, Faculty of Pharmaceutical Sciences, University of Sao Paulo, Sao Paulo, Brazil; ${ }^{2}$ Faculty of Pharmacy and Pharmaceutical Sciences, University of Alberta, Edmonton, Alberta, Canada

Received, June 25, 2015; Revised, October, 9, 2015; Accepted, October 9, 2015; Published, October 12, 2015.

ABSTRACT - The use of preservatives must be optimized in order to ensure the efficacy of an antimicrobial system as well as the product safety. Despite the wide variety of preservatives, the synergistic or antagonistic effects of their combinations are not well established and it is still an issue in the development of pharmaceutical and cosmetic products. The purpose of this paper was to establish a space design using a simplex-centroid approach to achieve the lowest effective concentration of 3 preservatives (methylparaben, propylparaben, and imidazolidinyl urea) and EDTA for an emulsion cosmetic product. Twenty-two formulae of emulsion differing only by imidazolidinyl urea (A: 0.00 to $0.30 \% \mathrm{w} / \mathrm{w}$ ), methylparaben (B: 0.00 to $0.20 \% \mathrm{w} / \mathrm{w}$ ), propylparaben (C: 0.00 to $0.10 \% \mathrm{w} / \mathrm{w}$ ) and EDTA (D: 0.00 to $0.10 \% \mathrm{w} / \mathrm{w}$ ) concentrations were prepared. They were tested alone and in binary, ternary and quaternary combinations. Aliquots of these formulae were inoculated with several microorganisms. An electrochemical method was used to determine microbial burden immediately after inoculation and after 2, 4, 8, 12, 24, 48, and $168 \mathrm{~h}$. An optimization strategy was used to obtain the concentrations of preservatives and EDTA resulting in a most effective preservative system of all microorganisms simultaneously. The use of preservatives and EDTA in combination has the advantage of exhibiting a potential synergistic effect against a wider spectrum of microorganisms. Based on graphic and optimization strategies, we proposed a new formula containing a quaternary combination (A: 55\%; B: 30\%; C: $5 \%$ and D: $10 \% \mathrm{w} / \mathrm{w}$ ), which complies with the specification of a conventional challenge test. A design space approach was successfully employed in the optimization of concentrations of preservatives and EDTA in an emulsion cosmetic product.

This article is open to POST-PUBLICATION REVIEW. Registered readers (see "For Readers") may comment by clicking on ABSTRACT on the issue's contents page.

\section{INTRODUCTION}

Preservatives are among the most controversial components of topical products. Consumer awareness and concerns towards hazards of these products are growing worldwide. The shift to safer products requires an innovative strategy to develop self-preserved systems and/or optimized concentration of these components in a formulation. The adoption of quality by design (QbD) allows achieving such goals. This systematic approach to development emphasizes product and process understanding using sound science and quality risk management (1). QbD primarily focuses on patient's safety by assessing critical quality attributes (CQA) and critical process parameters (CPP), which can have impact on the quality of the product. According to this framework, using a design space it is possible to demonstrate the relationship between factors affecting the CQA and CPP by a multi- dimensional combination and interaction of input variables (2).

The design of experiment (DoE), a multivariate systematic method, can be used to create a design space aiming to achieve an indepth knowledge and understanding of a formulation. Once approved, change within the limits established through a design space does not require regulatory approval (3). Experimental design has allowed choosing a combination of preservatives for specific targets during the development phase (4). The selected preservatives were $0.4 \%(\mathrm{w} / \mathrm{w})$ sorbic acid and $0.6 \%(\mathrm{w} / \mathrm{w})$ benzyl alcohol, which provided antimicrobial efficacy for a topic emulsion presenting high $\mathrm{pH}$.

Corresponding author: F.R. Lourenço (feliperl@usp.br) \& N. Bou-Chacra (chacra@usp.br), Department of Pharmacy, Faculty of Pharmaceutical Sciences, University of Sao Paulo, Av. Lineu Prestes 580, B1, Sao Paulo, Brazil. 
The approach showed the interaction among the selected preservatives, achieving a deeper understanding of their efficacy (4). Similarly, response surface methodology is applied to identify the optimal antimicrobial conditions of the natural peptides surfactin, polylysine and nisin against food spoilage organisms (5). Among the input variables, temperature, time exposure and preservative concentration, the latter is the most significant factor in the antimicrobial efficacy (5).

The ICH (2) points out that antimicrobial preservatives should be added in the product using the lowest effective concentration required to achieve efficacy throughout the intended shelf life of the product. The effectiveness in controlling the microbial growth is demonstrated by performing the antimicrobial preservative effectiveness test carried out during the development phase. The test allows comparing the antimicrobial activity of a variety of preservative systems in a pre-established condition; however, it presents some significant limitations as well. This test consists of challenging the formula against different microorganisms and enumeration of the survivors in specific time intervals using conventional pourplate methods (6). The major drawback of these classical microbial enumeration methods is rather a high workload and high variability. Therefore, these methods show low reproducibility. Thus, an alternative towards rapid methods and automation to enumerate microorganisms will enable the design space for the preservatives in a formulation.

According to Yang \& Bashir (7), the electrical method is the most successful of all the new introduced rapid methods in automation. This method is based on its ability to detect changes in the chemical composition of the culture media due to microbial growth, using electrical signals such as capacitance, conductance and impedance. During growth, microorganisms convert large molecules into small more highly charged molecules. Impedance, an electrical sign, associated with biosensor technology can reduce the assay time to between $30 \mathrm{~min}$ and $2 \mathrm{~h}$ compared with growth-based impedance method that takes at least $24 \mathrm{~h}$ to obtain the results. This approach renders this method even more interesting to be used in determining the burden in the preservative efficacy test in terms of obtaining faster results. Recently, Ferreira and collaborators (8) successfully replaced the conventional plate count method by the electrical one to determine the number of survivors in the preservative efficacy test of solid cosmetics. The study showed that the electrical method provided equivalent results to those obtained from the pour plate method for Pseudomonas aeruginosa, Staphylococcus aureus and Aspergillus brasiliensis.

In this study, the design space approach using a simplex-centroid design was applied aiming to achieve the lowest effective concentration of three preservatives, methylparaben, propylparaben, imidazolidinyl urea and EDTA, for an anti-aging eye fluid emulsion.

\section{MATERIALS AND METHODS}

\section{Materials}

The material is composed of 22 formulae of cream emulsion consisting of different preservatives and EDTA: imidazolidinyl urea (A: 0.00 to $0.30 \% \mathrm{w} / \mathrm{w}$ ), methylparaben (B: 0.00 to $0.20 \% \mathrm{w} / \mathrm{w}$ ), propylparaben $(\mathrm{C}: 0.00$ to $0.10 \%$ $\mathrm{w} / \mathrm{w}$ ) and EDTA (D: 0.00 to $0.10 \% \mathrm{w} / \mathrm{w}$ ) concentrations. They were tested alone and in binary, ternary and quaternary combinations (Figure 1). Cream emulsion formulae were composed of two phases: a lipophilic phase containing cetyl palmitate $(3.5 \% \mathrm{w} / \mathrm{w})$, isocetyl palmitate $(3.5 \% \mathrm{w} / \mathrm{w})$, cetyl alcohol $(0.75 \% \mathrm{w} / \mathrm{w})$, glyceryl stearate SE $(0.75 \% \mathrm{w} / \mathrm{w})$, steareth- 100 $(0.75 \% \mathrm{w} / \mathrm{w})$, ethyhexyl stearate $(0.75 \% \mathrm{w} / \mathrm{w})$, linoleic acid, linolenic acid $(2.0 \% \mathrm{w} / \mathrm{w})$, PEG-8 (and) tocopherol (and) ascorbyl palmitate (and) ascorbic acid (and) citric acid $(0.05 \% \mathrm{w} / \mathrm{w})$ and acrylates/C10-30 alkyl acrylate cross-polymer $(0.2 \% \mathrm{w} / \mathrm{w})$ as emulsifying agent; the hydrophilic phase consisted of butylene glycol $(5.0 \% \mathrm{w} / \mathrm{w})$, carbomer $(0.2 \% \mathrm{w} / \mathrm{w})$, hexylene glycol, fructose, glucose, sucrose, urea, dextrin, alanine, glutamic acid, aspartic acid, hexyl nicotinate $(3.0 \% \mathrm{w} / \mathrm{w})$, potassium hydroxide (q.s. $\mathrm{pH}$ 6.5) and purified water (QSP100\%). The preparation of this emulsion consisted of heating the two phases separately at a temperature of $75^{\circ} \mathrm{C}$. The lipophilic phase was added into the hydrophilic phase by stirring at $1,000 \mathrm{rpm}$ for 20 minutes. The preservatives were added after cooling to $40^{\circ} \mathrm{C}$.

Simplex-centroid design and statistical analysis A simplex-centroid mixture design was used to analyze the antimicrobial activity of three preservatives and EDTA (independent variables), as well as their interactions (synergism or antagonism). The feasible space for a mixture experiment with 4 variables is a pyramid presenting 4 vertices (one for each variable), called the simplex. The composition of each 


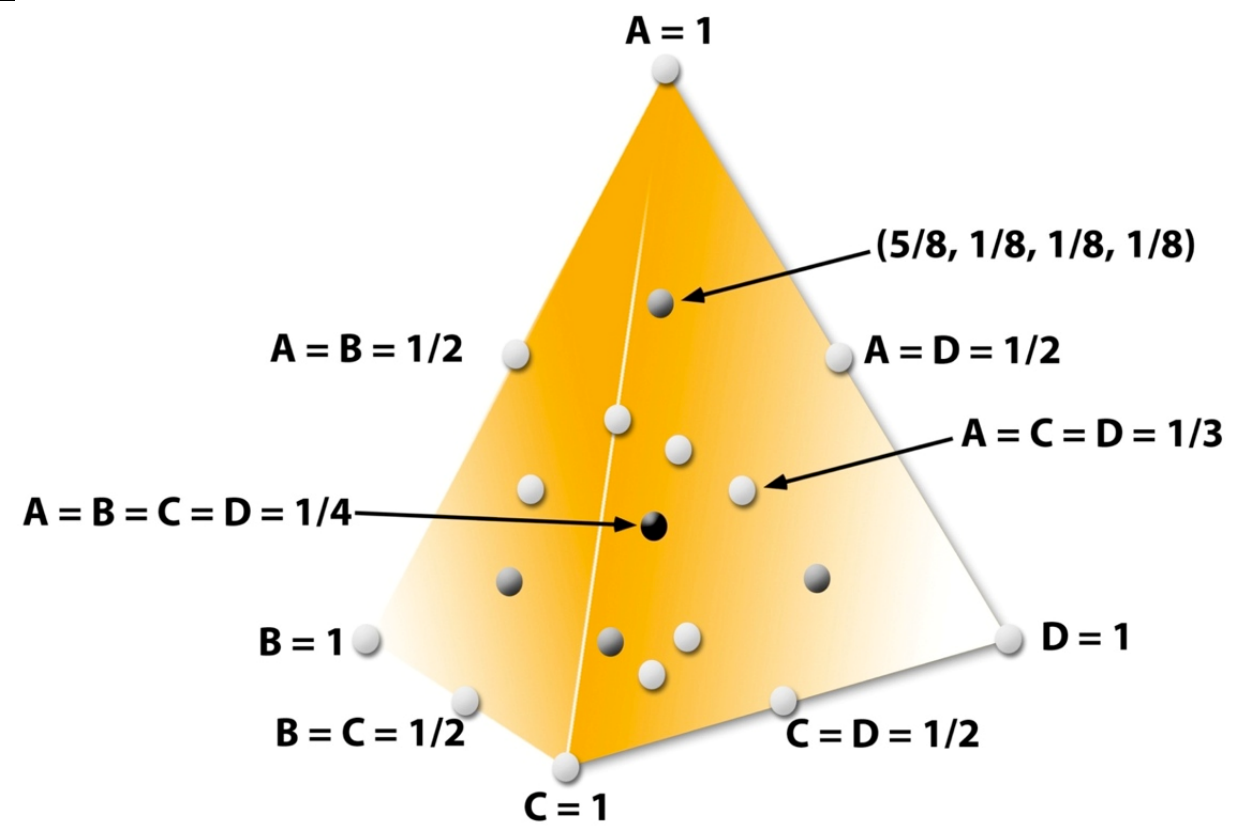

Figure 1. Binary, ternary and quaternary combinations of imidazolidinyl urea (A: 0.00 to $0.30 \mathrm{w} / \mathrm{w} \%$ ), methylparaben (B: 0.00 to $0.20 \mathrm{w} / \mathrm{w} \%$ ), propylparaben (C: 0.00 to $0.10 \mathrm{w} / \mathrm{w} \%$ ) and EDTA (D: 0.00 to $0.10 \mathrm{w} / \mathrm{w} \%$ ) concentrations in cream emulsion formulae.

mixture varies depending on its position on the simplex region, as presented in Figure 1. All the mixtures in the simplex must have the same final weight $(\mathrm{A}+\mathrm{B}+\mathrm{C}+\mathrm{D}=100)$. In the present work, 22 formulae containing a single component (preservative or EDTA) or their combinations were selected from the simplex. These formulae were provided by Minitab 17 (State College PA, USA) statistical software. The independent variables were imidazolidinyl urea (A); methylparaben (B); propylparaben (C), and EDTA (D), and their proportion combinations were presented in Table 1. The dependent variable or response was the angular coefficient (slope) of the resulting equation of linear regression curves by plotting the detection time versus time of inoculation. The curves were built for each formula and challenge microorganism, using the electrical method. When the microbial population reaches a threshold of $10^{6}-10^{7}$ cells $/ \mathrm{mL}$, an exponential change in the capacitance signal is observed. The time required for this change is called detection time and it is inversely proportional to the cell concentration. The angular coefficient (slope) reveals the microbiological property of the formulae. Positive and negative slopes indicated microbial death and microbial growth, respectively. This approach allows understanding the role of each independent variable alone and its binary, ternary or quaternary combination in the product development process, using statistical analysis.

\section{Optimization procedure}

The Minitab 17 statistical software (State College PA, USA) response optimizer tool was used to identify the combination of preservatives and EDTA proportions that provides increased antimicrobial activity against the challenge microorganisms. Response optimization was measured by the composite desirability, which allowed us to assess how well a combination of preservatives satisfies the antimicrobial activity against all tested microorganisms. Composite desirability ranges from zero to one. One represents the highest antimicrobial activity. On the other hand, zero indicates low or none antimicrobial activity.

\section{Model validation}

Based on optimization results, a new formula was proposed (Formula 23). Theoretically, formula 23 presents the lowest effective preservatives and EDTA concentrations against the challenge microorganisms. This formula was prepared and the preservative effectiveness was investigated using the conventional challenge test, as described in USP 37 (2014). 
Table 1. Mixture design with 3 preservatives and EDTA (A: imidazolidinyl urea; B: methylparaben; C: propylparaben; D: EDTA) as well as the angular coefficients (experimental responses - b) for Burkholderia cepacia (Bc), Pseudomonas aeruginosa $(\mathrm{Pa})$, Staphylococcus aureus $(\mathrm{Sa})$, Candida albicans $(\mathrm{Ca})$, and Aspergillus brasiliensis $(\mathrm{Ab})$.

\begin{tabular}{cccccccccc}
\hline Formula & $\mathbf{A ~ ( \% )}$ & $\mathbf{B ~ ( \% )}$ & $\mathbf{C ~}(\mathbf{\%})$ & $\mathbf{D}(\mathbf{\%})$ & $\mathbf{b B c}$ & $\mathbf{b P a}$ & $\mathbf{b S a}$ & $\mathbf{b C a}$ & $\mathbf{b A b}$ \\
\hline $1^{* 3}$ & 25.00 & 25.00 & 25.00 & 25.00 & 2.50 & 20.43 & 0.19 & 0.12 & 0.024 \\
$2^{* 2}$ & 50.00 & 50.00 & 0.00 & 0.00 & 1.38 & 2.28 & 0.36 & 0.096 & 0.96 \\
$3^{* 2}$ & 50.00 & 0.00 & 0.00 & 50.00 & 1.50 & 1.22 & 0.31 & 0.14 & 0.022 \\
$4^{* 2}$ & 12.50 & 12.50 & 62.50 & 12.50 & 1.06 & 1.54 & 0.09 & -0.34 & 2.05 \\
$5^{* 2}$ & 0.00 & 50.00 & 0.00 & 50.00 & 0.02 & 0.05 & 0.04 & 0.07 & 0.06 \\
$6^{* 1}$ & 100.00 & 0.00 & 0.00 & 0.00 & 5.23 & 3.79 & 1.14 & 0.00 & 7.29 \\
$7^{* 2}$ & 12.50 & 62.50 & 12.50 & 12.50 & 1.50 & 1.84 & 0.183 & -0.03 & 0.01 \\
$8^{* 2}$ & 50.00 & 0.00 & 50.00 & 0.00 & 2.51 & 3.84 & -0.05 & -0.04 & -0.02 \\
$9^{* 3}$ & 25.00 & 25.00 & 25.00 & 25.00 & 2.45 & 19.98 & 0.31 & 0.087 & 0.10 \\
$10^{* 2}$ & 12.50 & 12.50 & 12.50 & 62.50 & 1.39 & 0.83 & 0.25 & -0.50 & -0.04 \\
$11^{* 2}$ & 62.50 & 12.50 & 12.50 & 12.50 & 3.70 & 0.18 & 0.47 & 1.69 & $-0,26$ \\
$12^{* 1}$ & 0.00 & 100.00 & 0.00 & 0.00 & 0.26 & 0.03 & 0.01 & -0.05 & -0.05 \\
$13^{* 2}$ & 33.33 & 0.00 & 33.33 & 33.33 & 1.20 & 0.22 & 0.31 & 1.00 & -0.05 \\
$14^{* 2}$ & 33.33 & 33.33 & 0.00 & 33.33 & 2.96 & -0.30 & 0.33 & 0.07 & 0.05 \\
$15^{* 3}$ & 25.00 & 25.00 & 25.00 & 25.00 & 2.50 & 19.76 & 0.26 & 0.10 & -1.07 \\
$16^{* 2}$ & 0.00 & 50.00 & 50.00 & 0.00 & 0.21 & 0.00 & 0.26 & 0.05 & -0.06 \\
$17^{* 2}$ & 33.33 & 33.33 & 33.33 & 0.00 & 3.38 & 0.65 & 0.31 & -0.78 & 0.02 \\
$18^{* 2}$ & 0.00 & 33.33 & 33.33 & 33.33 & 0.24 & 0.50 & 0.31 & 0.06 & -0.02 \\
$19^{* 1}$ & 0.00 & 0.00 & 100.00 & 0.00 & -0.04 & 0.12 & -0.07 & -0.04 & 0.00 \\
$20^{* 1}$ & 0.00 & 0.00 & 0.00 & 100.00 & -0.04 & 24.00 & 0.03 & 0.03 & 0.00 \\
$21^{* 2}$ & 0.00 & 0.00 & 50.00 & 50.00 & 0.00 & 18.65 & 0.05 & 0.06 & -0.42 \\
$22^{* 3}$ & 25.00 & 25.00 & 25.00 & 25.00 & 2.54 & 18.23 & 0.21 & 0.08 & -0.42 \\
\hline
\end{tabular}

The independent variables are imidazolidinyl urea (A); methylparaben (B); propylparaben (C), and EDTA (D), and their proportion combinations represent the corners points ${ }^{* 1}$; mid points ${ }^{* 2}$, and center points ${ }^{* 3}$.

The dependent variables or responses are the angular coefficients (slopes) from the curves obtained by the microbial challenge using electrical method for each formula and microorganism.

\section{Preparation of inocula suspensions}

The challenge organisms were selected according to the general chapter $<51>$ Antimicrobial Effectiveness Testing (USP 37) for topic products. These organisms consisted of Pseudomonas aeruginosa (ATCC 9027) (Gramnegative bacillus), Staphylococcus aureus (ATCC 6538) (Gram-positive coccus), Candida albicans (ATCC 10231) (yeast) and Aspergillus brasiliensis (ATCC 16404) (mold). Burkholderia cepacia (ATCC 17759) (Gram-negative bacillus) was also selected in addition to the challenge organisms required in the official test (USP 37). Strains were obtained from the harvest of microbial growth of 24-hour-culture at $32 \pm 2.5^{\circ} \mathrm{C}$, using Tryptic Soy agar (TSA, Difco, Becton, Dickinson \& Cia, New Jersey, USA) for bacteria; 48 -hour-culture at $22 \pm 2.5^{\circ} \mathrm{C}$, using Sabouraud Dextrose agar (SDA, Difco, Becton, Dickinson \& Cia, New Jersey, USA) for yeast; and 7-day-culture at $22 \pm 2.5^{\circ} \mathrm{C}$, using SDA for the mold. Inocula suspensions were obtained using $5 \mathrm{~mL}$ of sodium chloride $(0.85 \% \mathrm{w} / \mathrm{v})$ for bacteria and yeast and sodium chloride $(0.85 \%$ $\mathrm{w} / \mathrm{v})$ with polysorbate $80(0.05 \% \mathrm{w} / \mathrm{v})$ for mold. The number of colony forming units $(\mathrm{CFU} / \mathrm{mL})$ of each suspension was determined by the pour-plate count method (USP 37). The period of incubation was $48 \mathrm{~h}$ for bacteria and yeast and $168 \mathrm{~h}$ for the mold. Inocula suspensions were diluted to provide $10^{7}-10^{8} \mathrm{CFU} / \mathrm{mL}$.

\section{Challenge test using electrical method}

Prior to challenge test, the formulae were submitted to the microbial limit test using the pour plate method (6). For the challenge test, the surviving organisms were determined by electrical capacitance signal using Bactometer equipment (Biolab-Mérieux, Marcy l'Etoile, France). This electrochemical method was performed by transferring portions of $50.0 \mathrm{~g}$ of each of 23 formulae (22 formulae of simplexcentroid mixture design and one optimized new formula - Formula 23) to sterile tubes, which were individually inoculated with $0.1 \mathrm{~mL}$ for each microbial suspensions, to obtained a burden of $10^{6}$ $\mathrm{CFU} / \mathrm{g}$. Ten-fold dilutions were made using TSA with polysorbate $20(0.7 \% \mathrm{w} / \mathrm{v})$ and soy lecithin $(0.1 \% \mathrm{w} / \mathrm{v})$ as neutralizers. Aliquots of $1.0 \mathrm{~mL}$ of dilutions 1:10 and 1:100 were transferred to wells containing $1.0 \mathrm{~mL}$ of General Purpose Medium (GPM-Plus, Biolab-Mérieux, Marcy l'Etoile, France) for bacteria and $1.0 \mathrm{~mL}$ of Yeast and 
Mold Medium (YMM, Biolab-Mérieux, Marcy l'Etoile, France), for yeast and mold. The wells were inserted into Bactometer, which was set to $48 \mathrm{~h}$ at $32 \pm 2.5^{\circ} \mathrm{C}$ for bacteria and $72 \mathrm{~h}$ at $22 \pm 2.5^{\circ} \mathrm{C}$, for the yeast and mold. Detection times range from 0 to $48 \mathrm{~h}$ for bacteria and 0 to $72 \mathrm{~h}$ for fungi, which represent the highest and the lowest bioburdens, respectively. Detection times (h) were determined immediately after inoculation the formula with the challenge microorganisms and after 2, 4, 8, 24 and $48 \mathrm{~h}$, for $P$. aeruginosa and $B$. cepacia; immediately after inoculation and after $2,4,12,24$ and $48 \mathrm{~h}$ for $S$. aureus; and immediately after inoculation and after 2, 4, 24, $48 \mathrm{~h}$ and $168 \mathrm{~h}$ for C. albicans and A. brasiliensis.

\section{Challenge test using the conventional plate method}

In addition to the electrical method, formula 23 (optimized formula) was also evaluated using conventional plate method to determine its microbiological property. Portions of $50.0 \mathrm{~g}$ of this formula were inoculated with $0.1 \mathrm{~mL}$ for each organism suspension to obtain a burden of $10^{6}$ $\mathrm{CFU} / \mathrm{g}$. The inoculated samples were maintained in glass bottles at room temperature. The sample inoculated with $B$. cepacia was aseptically removed after $0,2,4,8,24,48 \mathrm{~h}$ and 7, 14 and 28 days for viable counting. For P. aeruginosa, $S$. aureus and fungi, the viable counting was performed after $0,2,4,24$ and $48 \mathrm{~h}$ and 7, 14 and 28 days of inoculation.

The number of colony forming units $(\mathrm{CFU} / \mathrm{mL})$ of each sample was determined by the pour-plate count method (6). The first ten-fold dilution was performed using Tryptic Soy broth (Difco $\left.{ }^{\circledR}\right)$ with polysorbate $20(0.7 \% \mathrm{p} / \mathrm{v})$ for the bacteria and in Sabouraud Dextrose broth (Difco $\left.{ }^{\circledR}\right)$ with polysorbate $20(0.7 \% \mathrm{p} / \mathrm{v})$ for the fungi. The incubation time was $48 \mathrm{~h}$ at $32 \pm 2.5^{\circ} \mathrm{C}$ for bacteria and $72 \mathrm{~h}$ at $22.5 \pm 2.5{ }^{\circ} \mathrm{C}$, for the yeast and mold.

\section{RESULTS AND DISCUSSION}

\section{Challenge test by linear regression and} electrochemical method

Preliminary tests indicated no microbial contamination in all 23 formulae tested by conventional and electrochemical methods, which presented detection times above $48 \mathrm{~h}$. The samples complied with the microbial limit test $(<$ $10 \mathrm{CFU} / \mathrm{g}$ ) for the absence of pathogens (USP 37). Concerning the electrical signal, the capacitance was selected based on previous works indicating that this signal provides a better measurement of microbial growth than conductance for estimating the bacterial burden in surface water samples (9). Connoly and collaborators (10) and Zhou and King (11) also supported these findings by using the capacitance signal in their works for screening of cosmetic preservatives. Recently, Ferreira and collaborators (8) selected capacitance signal to evaluate the preservative efficacy of eyeshadow using freezedried organisms and an electrical method aiming to reduce the workload and the variability involved in PET for cosmetic powders. The electrical method is based on the measurement of changes due to growth and metabolic activity of the organisms in the culture medium. The bioburden is inversely correlated with the detection time (DT) (7), which is the time corresponding to a point at which the decrease in impedance value exceeds a threshold value usually when the organism number reaches approximately $10^{6}-10^{7} \mathrm{CFU} / \mathrm{mL}$. The plateau is established where the bacteria grow to a $10^{8}$ $\mathrm{CFU} / \mathrm{mL}$ or greater, and the depletion of medium components to be metabolized.

The estimate slopes were obtained from linear regression curves (detection time versus time of inoculation) as previous described. As the detection time is inversely proportional to the number of organism present at the sample, the negative slope values indicate microbial growth. On the other hand, the positive slope values indicate microbial death, which is a measure of antimicrobial activity of the preservative system. The slopes for all 22 formulae challenged using 5 microorganisms are presented in Table 1.

\section{Design space for preservatives and EDTA}

The slope values were used for the adjustment of equations to predict the effect of the concentrations of preservatives (A, B, and C) and EDTA (D) on the microbial burden. From the coefficients obtained for the independent variables (preservatives and EDTA concentrations), equations for each microorganism were generated, as presented in Table 2. The best fitting mathematical models were selected based on the comparisons of statistical parameters including the lack-of-fit adjustment ( $\mathrm{p}$-value), multiple correlation coefficient $\left(\mathrm{R}^{2}\right)$, adjusted multiple correlation coefficient (adjusted $\mathrm{R}^{2}$ ), and predicted correlation coefficient (predicted $\mathrm{R}^{2}$ ). The selected models were chosen considering the highest correlation coefficients as well as the evaluation for non-significant deviation ( $p$-value 
for lack-of-fit). These results are presented in Table 2 and Table 3.

Besides the microorganisms required by the official compendium (USP 37), Burkholderia cepacia was selected as a challenging organism due to its intrinsic preservative tolerance and remarkable adaptive mechanisms, such as multidrug resistance efflux pump. This strategy is an active transporter, which allows the organism pumping out the preservative when it reaches its cytoplasmic membrane (12). The regression analysis for $B$. cepacia resulted in a negative slope value for formulae 19 and 20 , both presenting value of -0.04 . (Table 1), which indicate microbial growth. Thus, no antimicrobial activity was observed for these formulae against this Gram-negative organism. Formulae 19 and 20 contain propylparaben and EDTA, respectively, as the single component (100\%).

Formulae 5, 12, 16 and 21 also presented lower or no antimicrobial activity against $B$. cepacia, with slopes equal to $0.02,0.26,0.21$, and 0.00 , respectively (Table 1). All these formulations $(5,12,16$ and 21) present the same missing component: imidazolidinyl urea. On the contrary, formulae 6 (slope equal to 5.23) and 17 (slope equal to 3.38), containing respectively imidazolidinyl urea alone and in combination with methyparaben and propylparaben, showed the highest antimicrobial activity for B. cepacia. Comparing the slopes for formulae 13,14, 17 and 18 containing a ternary combination of the preservatives and EDTA $(1 / 3 ; 1 / 3 ; 1 / 3)$, formulae 14 and 17 , containing imidazolidinyl urea and methylparaben presented the highest values: 2.96 and 3.38, respectively. Formula 13, containing imidazolidinyl urea but no methylparaben, presented a slope value of 1.20. Formula 18, without imidazolidinyl urea, presented the lowest slope: 0.24 . These results may indicate a synergic effect for the combination of imidazolidinyl urea and methylparaben. The slope values for the central formula (1, 9, 15 and 22), approximately 2.50 , support this finding. Thus, for B.cepacia, the best formulae are the ones containing only imidazolidinyl urea (Formula 6) and the formulae containing a binary combination of imidazolidinyl urea and methylparaben, using at least $25 \%$ of each preservative (Formulae 1, 9, 14, 15, 17 and 22). Considering the lower content of preservatives in these last formulations, compared to formula 6 , the central formula $(1 / 4 ; 1 / 4,1 / 4,1 / 4)$ can present effective antimicrobial property against this organism, according to the official criteria. Thus, by the design space approach, it was possible to identify the critical components (imidazolidinyl urea and methylparaben) whose levels (at least $25 \%$ in the formulation) have to be controlled to achieve preservative efficacy (Figure 2a). Furthermore, it was possible to characterize the interaction among the preservatives and EDTA.

Compared with the previous results, $P$. aeruginosa was more sensitive to the formulae than the other Gram-negative organism (range of slope between -0.30 and 24.00) (Table 1). The highest antimicrobial efficacy was obtained for formula 20 (slope equal to 24.00) containing $0.100 \%(w / v)$ of EDTA (Figure 2b).

Table 2. Predicted model for the angular coefficient (b) of the formulae containing different concentrations of 3 preservatives and EDTA for Burkholderia cepacia (Bc), Pseudomonas aeruginosa (Pa), Staphylococcus aureus (Sa), Candida albicans $(\mathrm{Ca})$, and Aspergillus brasiliensis $(\mathrm{Ab})$.

\begin{tabular}{|c|c|c|c|c|}
\hline Attribute & Predictive model & $R^{2}$ & $\begin{array}{c}R^{2} \\
(a d j)\end{array}$ & $\begin{array}{c}R^{2} \\
(\text { pred })\end{array}$ \\
\hline $\mathrm{bBc}$ & $\begin{array}{c}y=5.215 A+0.228 B-0.083 C+0.034 D-5.3938 A B-4.510 A D \\
+55.170 A B C+58.868 A B D\end{array}$ & $99.84 \%$ & $98.44 \%$ & $99.75 \%$ \\
\hline $\mathrm{bPa}$ & $\begin{aligned} y=4.4 A-0.4 B & +0.5 C+23.9 D-50.1 A D-45.3 B D+26.4 C D \\
& -179.9 A C D-160.4 A C(A-C)-471.7 B^{2} C D \\
& -693.6 C D(C-D)+5474.1 A B C D\end{aligned}$ & $99.46 \%$ & $98.87 \%$ & $86.62 \%$ \\
\hline $\mathrm{bSa}$ & $\begin{array}{c}y=1.15 A+0.01 B-0.07 C+0.03 D-0.84 A B-2.30 A C-1.08 A D \\
+1.14 B+11.80 A B^{2} D+13.53 A B C^{2}+22.52 A C D^{2} \\
+12.33 B C D^{2}-25.30 A B C D\end{array}$ & $99.17 \%$ & $97.82 \%$ & $88.83 \%$ \\
\hline $\mathrm{bCa}$ & $\begin{aligned} y=-0.03 B- & 0.04 C+0.05 D+0.40 A B+0.42 A D+0.35 B C \\
& +0.23 C D-22.97 A B C+24.68 A C D \\
& -2.05 A B(A-B)+30.87 A D(A-D) \\
& -10.45 C D(C-D)\end{aligned}$ & $99.78 \%$ & $99.48 \%$ & $97.62 \%$ \\
\hline $\mathrm{bAb}$ & $\begin{array}{c}y=7.27 A+0.06 B-0.09 C-0.04 D-10.19 A B-13.79 A C \\
-14.22 A D+10.63 A B D+19.95 A C D \\
-58.25 A C(A-C)\end{array}$ & $98.90 \%$ & $98.08 \%$ & $92.30 \%$ \\
\hline
\end{tabular}


Table 3. Analysis of variance for the different models' fitted-responses (b) (Table 1) for Burkholderia cepacia (Bc), Pseudomonas aeruginosa (Pa), Staphylococcus aureus (Sa), Candida albicans (Ca), and Aspergillus brasiliensis (Ab).

\begin{tabular}{|c|c|c|c|c|c|c|c|c|c|c|c|c|c|c|c|}
\hline & \multicolumn{3}{|c|}{ bBC } & \multicolumn{3}{|c|}{$\mathbf{b P a}$} & \multicolumn{3}{|c|}{ bSa } & \multicolumn{3}{|c|}{ bCa } & \multicolumn{3}{|c|}{ bAdb } \\
\hline Source & $\mathrm{df}$ & $\begin{array}{l}\text { Seq } \\
\text { SS }\end{array}$ & $\mathrm{P}$ & $\mathrm{df}$ & $\begin{array}{l}\text { Seq } \\
\text { SS }\end{array}$ & $\mathrm{P}$ & $\mathrm{df}$ & $\begin{array}{l}\text { Seq } \\
\text { SS }\end{array}$ & $\mathrm{P}$ & $\mathrm{df}$ & $\begin{array}{l}\text { Seq } \\
\text { SS }\end{array}$ & $\mathrm{P}$ & $\mathrm{df}$ & $\begin{array}{l}\text { Seq } \\
\text { SS }\end{array}$ & $\mathrm{P}$ \\
\hline Regression & 8 & 43.125 & 0.000 & 11 & 1633.7 & 0.000 & 13 & 1.280 & 0.000 & 12 & 3.48 & 0.000 & 9 & 54.622 & 0.000 \\
\hline Linear & 3 & 31.969 & 0.000 & 3 & 320.40 & 0.000 & 3 & 0.815 & 0.000 & 3 & 0.322 & 0.148 & 3 & 21.779 & 0.000 \\
\hline Quadratic & 3 & 1.287 & 0.000 & 3 & 173.10 & 0.000 & 5 & 0.376 & 0.000 & 4 & 0.342 & 0.009 & 3 & 23.185 & 0.000 \\
\hline $\begin{array}{l}\text { Special } \\
\text { cubic }\end{array}$ & 2 & 9.868 & 0.000 & 1 & 80.74 & 0.000 & - & - & - & 2 & 1.027 & 0.000 & 2 & 0.738 & 0.000 \\
\hline Full cubic & - & - & - & 1 & 0.26 & 0.000 & - & - & - & 1 & 8.920 & 0.000 & 1 & 6.733 & 0.000 \\
\hline $\begin{array}{l}\text { Special } \\
\text { quartic }\end{array}$ & - & - & - & 1 & 46.68 & 0.000 & 4 & 0.073 & 0.001 & - & - & - & - & - & - \\
\hline Full quartic & - & - & - & 1 & 183.20 & 0.000 & 1 & 0.015 & 0.009 & - & - & - & - & - & - \\
\hline Lack of fit & 10 & 0.064 & 0.114 & 7 & 6.11 & 0.570 & 5 & 0.002 & 0.965 & 6 & 0.007 & 0.138 & 9 & 0.455 & 0.557 \\
\hline
\end{tabular}

This antimicrobial activity was 4.6 times higher compared with the best activity against $B$. cepacia (formula 6 slope equal to 5.23). EDTA is frequently associated with several preservatives since it enhances their antimicrobial activity (13). It is a common ingredient in cosmetics used as stabilizer by binding metal ions, which can cause oxidative degradation of the active components. Besides, the formulation presents carbopol 940, a cross-linked polyacrylate polymer. It is an extremely efficient rheology modifier capable of providing high viscosity and forms hydroalcoholic gels and creams. Scalzo and collaborators (14) showed that this polymer exerted an interesting synergism in antimicrobial activity of the preserving agent against $P$. aeruginosa. According to the authors (14), a concentration equal to $0.2 \%(\mathrm{w} / \mathrm{w})$ of carbopol showed intense antimicrobial activity against $P$. aeruginosa associated with methylparaben. The mechanism involved in the antimicrobial activity of carbopol was similar to those observed for the EDTA. This potent chelating agent removes stabilizing cations from the outer membrane of Gram-negative organisms. This membrane provides a challenging barrier that must be overcome. The permeability properties of this barrier reduce significantly the susceptibility of the organism to antimicrobials, which are targeted at intracellular processes (15). According to our results, the predicted model $\left(\mathrm{R}^{2}=0.995, \mathrm{R}^{2}\right.$ adj $=$ $0.989, \mathrm{R}^{2}$ pred 0.866 , lack-of-fit equal to 0.570 , $\alpha=0.05$ ) (Table 2 and Table 3) indicates that the combination of imidazolidinyl urea, methylparaben propylparaben and EDTA showed a synergistic effect (Formulae 1, 9, 15 and 22) against $P$. aeruginosa. On the other hand, the combination of imidazolidinyl urea and EDTA (Formula 3, slope value equal to 1.22) and the combination of methylparaben and EDTA (Formula 5, slope equal to 0.05) showed an antagonist effect against $P$. aeruginosa. Similar to the previous results using $B$. cepacia, the central formula showed the best performance aiming to achieve the lowest possible concentration of the preservatives and EDTA presenting antimicrobial efficacy.

On the contrary, the formulae presented lower antimicrobial activity against $S$. aureus and $C$. albicans comparing to the Gram-negative organisms. The carbopol, presented in the formulation, depressed the antimicrobial effect of the preservatives against these organisms by chemical or physical interaction of preservative with this macromolecule. Furthermore, it is possible that the hydrocolloid provided physical protection for microbial cells (14). In this study, among the bacteria, $S$. aureus presented the highest resistance to the formulae, consistent with findings by Scalzo and collaborators (14). The highest antimicrobial activity was observed for formula 6 (slope equal to 1.14), which contains $0.3 \%(\mathrm{w} / \mathrm{w})$ of imidazolidinyl urea (Table 1). Formula 11 showed a slope of 0.47 , the second highest value. The predict model $\left(\mathrm{R}^{2}=99.17 \%\right.$, $\mathrm{R}^{2}$ adj $=97.82 \%, \mathrm{R}^{2}$ pred $88.83 \%$, lack-of-fit equal to 0.965, $\alpha=0.05$ ) (Table 2 and Table 3) indicated that imidazolidinyl urea is the critical component for the antimicrobial preservative efficacy of the formula (Figure 2c). The central formula $(1,9,15$ and 22) presented average slope values of 0.24 .

The highest antimicrobial activity for $C$. albicans was observed for formula 11 (slope equal to 1.69$)$, containing $62.5 \% \quad(\mathrm{w} / \mathrm{w})$ of imidazolidinyl urea, $12.5 \% \quad(\mathrm{w} / \mathrm{w})$ of methylparaben, $12.5 \%(\mathrm{w} / \mathrm{w})$ of propylparaben and $12.5 \%(\mathrm{w} / \mathrm{w})$ of EDTA (Table 1). 

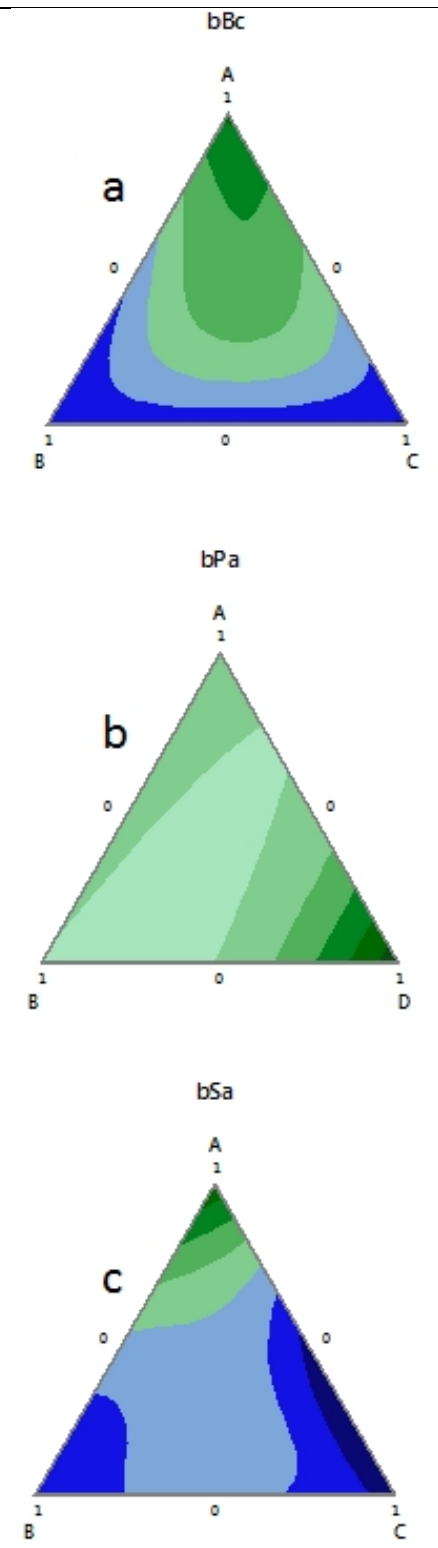
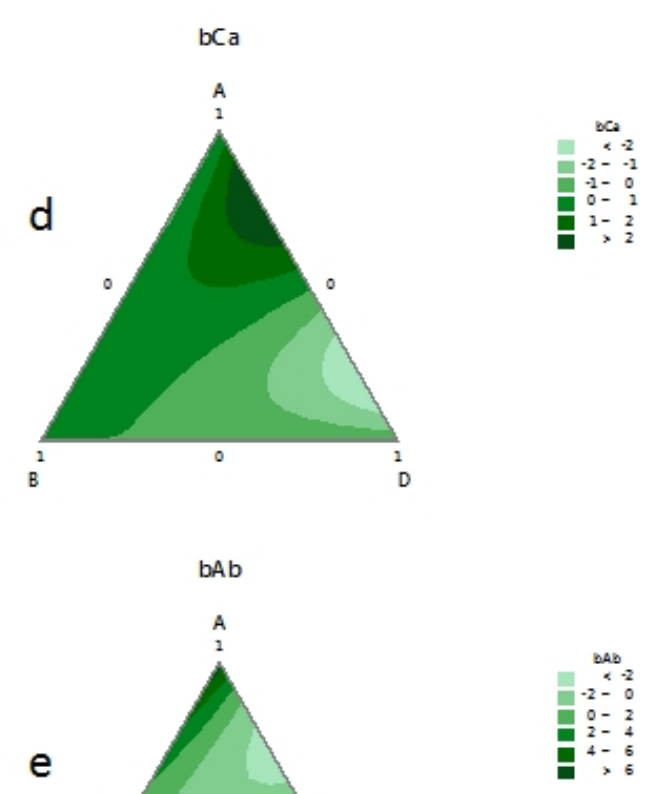

Figure 2: Contour plots for the effect of different combinations of preservative and EDTA on angular coefficients (b) for Burkholderia cepacia (Bc), Pseudomonas aeruginosa (Pa), Staphylococcus aureus (Sa), Candida albicans (Ca), and Aspergillus brasiliensis (Ab).

This result compared to those from formulae 6 , 12,19 and 20 , slope values equal to $0,-0.05$, 0.07 and 0.032 , containing a single component (imidazolidinyl urea or methylparaben or propylparaben or EDTA) respectively, may indicate a synergistic effect of imidazolidinyl urea, propylparaben and EDTA against the yeast. The predict model $\left(\mathrm{R}^{2}=99.78 \%, \mathrm{R}^{2}\right.$ adj $=$ $99.48 \%, \mathrm{R}^{2}$ pred $97.62 \%$, lack-of-fit equal to 0.138, $\alpha=0.05$ ) (Table 2 and Table 3) indicated that the quaternary combination of preservatives and EDTA containing imidazolidinyl urea at concentration of $62.5 \%(\mathrm{w} / \mathrm{w})$ is critical for the antimicrobial preservative efficacy of the formula (Figure 2d). Formula 13, a ternary combination of imidazolidinyl urea, propylparaben and EDTA, presented a slope value of 1.00 , corroborating this finding. All other formulae presented slope values lower than 0.14 (reduced antimicrobial activity) or negative slopes (microbial growth).

Regarding the mold, it presented the highest antimicrobial resistance to the formulae compared to the other organisms, with few exceptions. Formula 6 (slope equal to 7.29), containing imidazolidinyl urea as the single component $(100 \% \mathrm{w} / \mathrm{w})$ showed the higher antimicrobial 
activity. The predictive model $\left(\mathrm{R}^{2}=98.90 \%, \mathrm{R}^{2}\right.$ adj $=98.08 \%, R^{2}$ prev $92.30 \%$, lack-of-fit equal to 0.557, $\alpha=0.05$ ) (Table 2 and Table 3) confirmed that this component is critical for the antimicrobial preservative efficacy of the formula (Figure 2e). Formula 2, a binary combination containing $50 \%(\mathrm{w} / \mathrm{w})$ of imidazolidinyl urea and $50 \%$ of methylparaben and Formula 4, a quaternary combination containing $12.5 \%(\mathrm{w} / \mathrm{w})$ of imidazolidinyl urea, $12.5 \% \quad(\mathrm{w} / \mathrm{w})$ of methylparaben, $62.5 \%(\mathrm{w} / \mathrm{w})$ of propylparaben and $12.5 \%(\mathrm{w} / \mathrm{w})$ of EDTA presented slope values of 0.96 and 2.05 , respectively. All the remaining formulae showed very low antimicrobial activity (slope close to zero) against this organism or microbial growth (negative slope).

\section{Optimization procedure}

Imidazolidinyl urea in combination with parabens is one of the most widely used preservative systems, commonly found in cosmetics. Parabens alone provide inadequate protection against Pseudomonas growth in personal care products. The addition of imidazolidinyl urea to these products is shown to enhance its spectrum action against Pseudomonas, a metabolically versatile organism (16), known as the nemesis of cosmetic industry (17).

The response optimizer tool revealed the concentrations of imidazolidinyl urea, parabens and EDTA that resulted in a most effective preservative system against all tested microorganisms simultaneously. An excellent outcome was reached (desirability=1.0) (Table 4 and Figure 3). The formula containing a quaternary combination of imidazolidinyl urea $(55 \% \mathrm{w} / \mathrm{w})$, methylparaben $(30 \% \mathrm{w} / \mathrm{w})$, propylparaben $(5 \% \mathrm{w} / \mathrm{w})$, and EDTA $(10 \% \mathrm{w} / \mathrm{w})$ (formula 23) was the most effective against the challenge microorganisms. This formula showed similar predicted and observed slope values for the challenge organisms (Table 5). This result validated the proposed model. It was possible to determine the limits in which the product can meet the criteria for the preservative efficacy. In addition, formula 23 met the official criteria (Table 6), for the antimicrobial preservative efficacy test.

Table 4. The desirable ranges for each response (b) in the optimization procedure for Burkholderia cepacia (Bc), Pseudomonas aeruginosa (Pa), Staphylococcus aureus (Sa), Candida albicans (Ca), and Aspergillus brasiliensis

\begin{tabular}{cccc}
$(\mathrm{Ab})$. & & & \\
\hline Parameters & Goal & Lower & Target \\
\hline $\mathrm{bBc}$ & Maximum & 0.1 & 2.0 \\
$\mathrm{bPa}$ & Maximum & 0.1 & 2.0 \\
$\mathrm{bSa}$ & Maximum & 0.1 & 0.3 \\
$\mathrm{bCa}$ & Maximum & 0.1 & 0.3 \\
$\mathrm{bAb}$ & Maximum & 0.1 & 0.3 \\
\hline
\end{tabular}

Table 5. Predicted and observed slope values (b) obtained for formula 23 for Burkholderia cepacia (Bc), Pseudomonas aeruginosa (Pa), Staphylococcus aureus (Sa), Candida albicans (Ca), and Aspergillus brasiliensis $(\mathrm{Ab})$.

\begin{tabular}{ccc}
\hline Microorganism & Predicted slope value (b) & Observed slope value (b) \\
\hline $\mathrm{Bc}$ & 3.25 & 3.40 \\
$\mathrm{~Pa}$ & 2.40 & 2.10 \\
$\mathrm{Sa}$ & 0.44 & 0.51 \\
$\mathrm{Ca}$ & 0.42 & 0.42 \\
$\mathrm{An}$ & 0.57 & 0.55 \\
\hline
\end{tabular}

Table 6. Antimicrobial effectiveness testing for Formula 23.

\begin{tabular}{lccccccc}
\hline \multirow{2}{*}{ Microorganism } & \multicolumn{7}{c}{ Time intervals (h) } \\
\cline { 2 - 8 } & T0 & T2 & T4 & T8 & T24 & T48 & T168 \\
\hline B. cepacia & 5.45 & 4.33 & 3.87 & - & - & - & - \\
P. aeruginosa & 5.80 & 5.00 & 4.64 & $*$ & - & - & - \\
S. aureus & 5.26 & 5.40 & 5.08 & $*$ & 5.08 & 4.91 & - \\
C. albicans & 4.29 & 4.62 & 3.97 & $*$ & 2.42 & 1.71 & - \\
A. brasiliensis & 4.93 & 4.58 & 4.02 & $*$ & 2.79 & 0.74 & - \\
\hline
\end{tabular}

$(-)<10 \mathrm{CFU} / \mathrm{g} /\left(^{*}\right)$ not performed. T14 days and T28 days: $<10 \mathrm{CFU} / \mathrm{g}$.

Required criteria for antimicrobial effectiveness (USP 37): not less than 2.0 log reduction from the initial count at 14 days and no increase from the 14 days' count at 28 days, for bacteria; no increase from the initial calculated count at 14 and 28 days, for the yeast and mold. 


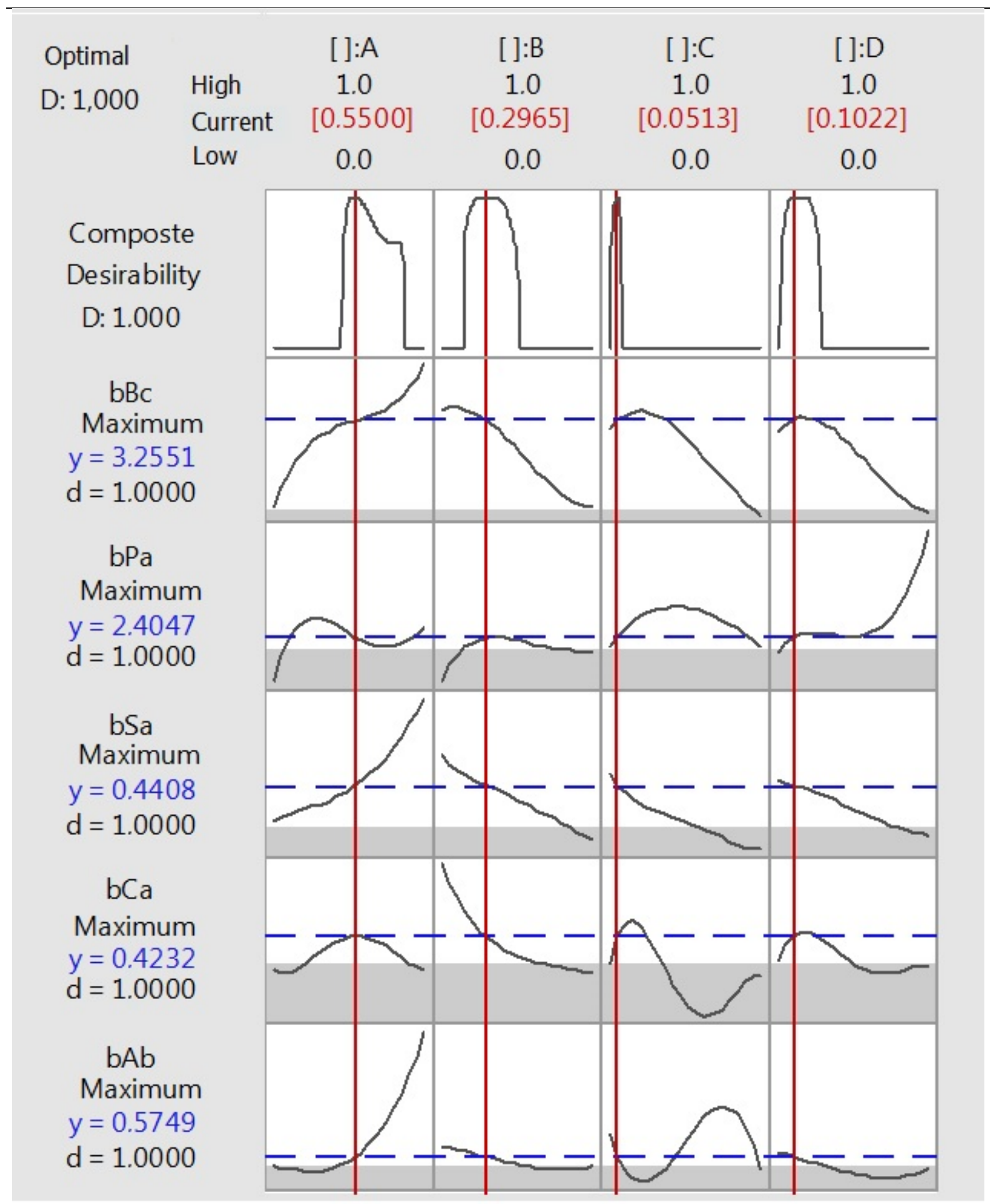

Figure 3: Optimization plots for the effect of different combinations of preservative and EDTA on angular coefficients (b) for Burkholderia cepacia (Bc), Pseudomonas aeruginosa (Pa), Staphylococcus aureus (Sa), Candida albicans (Ca), and Aspergillus brasiliensis (Ab).

The use of preservatives and EDTA in combination has the advantage of exhibiting a potential synergistic effect against a wider spectrum of microorganisms. The determination of the minimum inhibitory concentration showed that the efficacy of the preservative system could markedly lower the concentration of phenoxyethanol when used in combination with 
other preservatives (18). Their result is consistent with those obtained in this study, confirming the theory that the use of a preservative combination can result in better protection of the formula.

\section{CONCLUSION}

The design space approach was successfully employed in the optimization of concentrations of preservatives (methylparaben, propylparaben and imidazolidinyl urea) and EDTA regarding their effectiveness against B. cepacia (ATCC 17759), $P$. aeruginosa (ATCC 9027), S. aureus (ATCC 6538), C. albicans (ATCC10231) and $A$. brasiliensis (ATCC16404). This approach allowed describing the antimicrobial performance of the formula against the organisms as a mathematical function of the preservatives and EDTA concentration. Furthermore, it was possible to identify the critical components and the limits in which variations in their concentrations have minimal or no effect in the antimicrobial activity. In addition, this study allowed identifying the interactions among the preservatives and EDTA. According to the optimization results, the formula containing a quaternary combination of imidazolidinyl urea $(55 \% \quad \mathrm{w} / \mathrm{w})$, methylparaben $(30 \% \mathrm{w} / \mathrm{w})$, propylparaben $(5 \% \mathrm{w} / \mathrm{w})$ and EDTA $(10 \% \mathrm{w} / \mathrm{w})$ was the most effective against all microorganisms, simultaneously. This formula reduced the microbial burden before $48 \mathrm{~h}$. The use of the electrical method allowed a comprehensive evaluation of the antimicrobial property of an anti-aging eye fluid emulsion by reducing the workload and turnaround time inherent in the conventional antimicrobial preservative efficacy test.

\section{REFERENCES:}

1. Yu, LX. Gregory A, Khan MA, Hoag, SW, Polli, J, Raju, GK, Woodcock, J. Understanding pharmaceutical quality by design AAPS J, 2014; 16(4): 771-783.

2. INTERNATIONAL CONFERENCE ON HARMONISATION OF TECHNICAL REQUIREMENTS FOR REGISTRATION OF PHARMACEUTICALS FOR HUMAN USE. Work Products. ICH Guidelines. Quality Guidelines. Q8 Pharmaceutical Development Q8 (R2) (Accessed May 5, 2015, http://www.ich.org/fileadmin/Public Web Site/ICH Products/Guidelines/Quality/ Q8 R1/Step4/Q8 R2 Guideline.pdf).

3. Patil AS, Pethe, AM. Quality by Design (bD): a new concept for development of quality pharmaceuticals Int J Pharm Quality Assurance,
2013; 4(2): 13-19

4. Rahali, Y, Pensé-Lhéritier, AM, Mielcareck, C, Bensouda, Y. Optimization of preservatives in a topical formulation using experimental design Int J Cosmetic Sci, 2009; 31: 451-460.

5. Huang, X, Li, M, Zhao, G, Gao, X, Zhang, Q, Sun, L, Liu, Y, Xia W. Optimization on antimicrobial effects of natural compound preservative against $B$. cereus and E. coli by RMS Int J Pept Res Ther, 2012; 18: 383-389.

6. United States Pharmacopeia, 2014. USP 37. United States Pharmacopoeial Convention, Rockville.

7. Yang, L, Bashir, R. Electrical/electrochemical impedance for rapid detection of foodborne pathogenic bacteria Biotechnol Adv,2008; 26:135-150.

8. Ferreira, MRS, Lourenço, FR, Ohara, MT, BouChacra, NA, Pinto, TJA. An innovative challenge test for solid cosmetics using freeze-dried microorganisms and electrical methods $\mathrm{J}$ Microbiol Meth, 2014; 106(1): 104-109.

9. Noble, PS, Dziuba, M, Harrison, DJ, Albritton, WL. Factors influencing capacitance-based monitoring of microbial growth J Microbiol Meth, 1999; 37: 51-64.

10. Connolly, P, Bloomfield, SF, Denyer, SP. The use of impedance for preservative efficacy testing of pharmaceuticals and cosmetics J Appl Bacteriol, 1994;76: 68-74.

11. Zhou, X, King, VM An impedimetric method for rapid screening of cosmetic preservatives. J Ind Microbiol, 1995; 15: 103-107

12. Rushton, L, Sass, A, Baldwin, A, Dowson, CG, Donoghue, D, Mahenthiralingama, E. Key role for efflux in the preservative susceptibility and adaptive resistance of Burkholderia cepacia complex bacteria. Antimicrob Agents Ch, 2013; 57(7): 2972-2980.

13. Lambert, RJW, Hanlon, GW, Denyer, SP. The synergistic effect of EDTA/antimicrobial combinations on Pseudomonas aeruginosa J Appl Microbiol, 2004;. 96: 244-253.

14. Scalzo, M, Orlandi, C, Simonetti, N, Cerreto, F. Study of interaction effects of polyacrylic acid polymers (carbopol 940) on antimicrobial activity of methylparahydroxybenzoate against some gram-negative, gram-positive bacteria and yeast $\mathrm{J}$ Pharm Pharmacol, 1996; 48: 1201-1205.

15. Ferris, FG. Metallic ion interactions with the outer membrane of gram-negative bacteria. In: Beveridge, TJ, Ronald, JD (eds) Metal ions and bacteria. John Wiley \& Sons (ed), 1989: 302-305.

16. Berke, PA, Rosen, WE. Imidazolidinyl urea activity against Pseudomonas. J Soc Cosmet Chem, 1978, 29:757-766.

17. Orth, D. Insights into cosmetic microbiology, Allured Business Media (ed), 2010: 263-267, $350 \mathrm{p}$.

18. Lundov, MD, Johansen, JD, Zachariae, C, Moesby, L. Low-level efficacy of cosmetic preservative Int J Cosm Sci, 2011; 33: 190-196. 